\title{
THE EFFECT OF CULTURAL TRANSMISSION ON CONTINUOUS VARIATION
}

\author{
LINDON EAVES \\ Department of Genetics, University of Birmingham, Birmingham B15 2TT
}

Received 12.xi.75

\begin{abstract}
SUMmaRY
Cultural transmission may depend on the non-genetic transfer of information from parent to offspring. The consequences of such cultural transmission for continuous variation are investigated theoretically for randomly mating populations. Cultural inheritance may act on genetical and environmental differences between individuals. The consequences for cultural inheritance of polygenic variation and variation due to chance environmental factors are considered. An equilibrium may occur in which the population variance and the covariances between relatives can be expressed as functions of estimable parameters of genetical and environmental variation. Whatever the ultimate origin of culturally inherited differences they are expected to lead to environmental differences between families (" $E_{2}$ " variation). In addition, if cultural transmission maintains differences due ultimately to segregation at many gene loci we may find genotype-environmental covariation is generated.
\end{abstract}

\section{INTRODUCTION}

THE application of biometrical genetics to the analysis of human behaviour has revived interest in the role of cultural transmission in maintaining human variation. Models which assume some genetical variation have given a fairly satisfactory account of individual differences in intelligence (e.g. Jinks and Fulker, 1970; Eaves, 1973, 1975; Jencks, 1973), personality (Eaves and Eysenck, 1975) and even in social attitudes (Eaves and Eysenck, 1974; Hewitt, 1974; Martin, 1975 pers. comm.). Certain unease has been expressed about the data to which such models have been fitted, especially with regard to intelligence (e.g. Jensen, 1974). In the case of personality and social attitudes genotype-environmental models have only really been tested on twin data so the collection of data on other kinds of relatives is desirable in order that the adequacy of such explanations can be studied more generally. As far as can be judged from data analysed so far, however, a fairly simple model of gene action and environmental causation could account for the bulk of measured variation.

In testing these simple genotype-environment models a few equally simple environmental models have been shown to offer less satisfactory explanations of the available data. So far, there are few more subtle treatments of environmental causation which can claim to be anything better than ad hoc rationalisations of particular sets of data. A general quantitative theory of environmental variation is required if environmental explanations of human variation are to compete seriously with the genotype-environmental models explored so far. Failure to provide such a quantitative theory can only weaken any claim to serious attention of a purely environmental explanation of individual differences. 
There are, however, several kinds of environmental variation which can be expressed in the form of a quantitative model. The formulation of such models is instructive because it is necessary to be quite precise about the nature of assumptions which can easily be glossed over in a merely verbal discussion of the problem. Furthermore, a precisely formulated model provides a sound basis for deciding what data need be collected in order to test the assumptions it implies and to estimate the relevant parameters of an adequate model.

In this paper we examine some consequences of cultural transmission for the maintenance of continuous variation and specify certain of its effects on the covariation between relatives.

Before any model can be formulated, we have to be quite explicit about the assumed origin of cultural variation and the mechanism by which it is perpetuated. Verbal explanations of variation in cultural terms sometimes fail to make this distinction. The mechanism of cultural transmission, whether it be spoken and written language or other human interactions, does not create diversity, it maintains amplifies or dampens it. Cultural transmission relates to cultural variation just as hereditary transmission relates to genetical variation. The existence of a mechanism for cultural transmission does not answer the other fundamental question of the origins of those differences which culture ameliorates or exaggerates.

Ultimately, variation maintained by cultural transmission is unlikely to differ from any other kind of phenotypic variation. It should be traceable to the action and interaction of hereditary and environmental factors. Any model of cultural variation which takes no account of the possible biological basis of variation is likely to have little predictive value. Human populations are genetically heterogeneous and it is naïve to suppose that this heterogeneity is without consequences in the cultural as well as the physical domain. It would be equally naïve to suppose, a priori, in a species where the behaviour of one individual is able to influence the behaviour of another, that such cultural transmission is without significance for variation in particular human traits. The treatment of cultural transmission in this paper, albeit open to criticism on grounds of simplicity, will show that the pattern of variation and covariation between relatives will depend on what factors are responsible for the origin of culturally transmissible differences. We shall explore the quantitative consequences of the cultural transmission of differences which arise in two different sources: chance environmental variation and segregation of many gene loci of small individual effects. Neither genes nor environment, individually, are likely to provide an adequate account of variation. However, having specified in a model the effects of genes and environment separately we may, in certain circumstances, simply combine the two sets of expectations to obtain those which apply when cultural transmission perpetuates differences initially due to the joint additive and independent effects of genetic segregation and random environmental factors.

As far as the mechanism of cultural transmission is concerned we shall, for the purposes of developing our particular treatment, follow the suggestion of Cavalli-Sforza and Feldman (1973) who assume for the sake of model building that the cultural environment of offspring depends largely on the phenotypes of their parents for the trait measured. Cultural factors which are independent of the measured aspect of the parental phenotype will have 
rather different consequences which can often be represented by somewhat simpler models than those we consider here. We choose to concentrate on the cultural influence of the parental phenotype because of its substantial theoretical interest in human biology and because of its possible relevance to identifying major sources of culturally maintained inequality. Parents may influence their children in a variety of ways, either directly through their use of language or other means, or indirectly by virtue of the social and educational facilities of the area in which the parents live whether by choice or circumstance. Detecting and analysing the parental basis of cultural transmission, therefore, may be of primary importance for our understanding of any cultural basis of human variation.

Even within this restricted aim, there might exist many variations on the theme of parental influence. One or other parent may play the major cultural role, or both may be equally responsible for the quality of the environment in which their offspring develop. The model we shall develop here assumes that both parental phenotypes are culturally significant. Models which assume only one parent is involved are easier to handle analytically, those which allow for unequal contributions from the two parents are rather more tedious to develop but not intractable.

Up to a point we can formulate the basic concepts of our model without committing ourselves to any particular explanation, genetical or environmental, of the origin of culturally transmitted differences.

\section{The LINEAR MODEL AND THE EXPEGTED POPUlation VARIANCE}

First we introduce the notation. We shall number generations backwards from the current generation to which we shall refer as "generation $0 "$ ".

We let the phenotypic score of the $i$ th individual in the current generation $(n=0)$ be $p_{0 i}$. In the $n$th ancestral generation individual $i$ has $2^{n}$ ancestors. The phenotype of the $j$ th ancestor in the $n$th generation will be $p_{n i j}$. In the absence of cultural transmission the phenotype of an individual in generation $n$ can be written without reference to his ancestors in the form:

$$
p_{n i}=\xi_{n}+x_{n i}
$$

$\xi_{n}$ is the mean effect of all influences genetic or environmental, but not cultural, which contribute to variation in $p_{n}$. We let $x_{n i}$ denote the noncultural effect of the $i$ th individual, expressed as a deviation from $\xi_{n}$. " Noncultural effects" refer to any effects contributing to individual differences which are not the direct result of the cultural impact of the parental phenotype. Non-cultural effects may be genetic or environmental; probably both. If they are genetic, then the non-cultural effects of offspring are expected to covary with the cultural effects they receive from their parents.

If the parental phenotype exercises a cultural impact on the phenotype of offspring we may write, for the phenotype of individual $i$ in generation $n$,

$$
p_{n i}=\xi_{n}+x_{n i}+b \sum_{j=1}^{j=2} p_{n+1, i j}
$$

generation $n+1$ being one generation further back than generation $n$.

The coefficient $b$ represents that multiple of the phenotype of a parent 
which is expressed culturally in the phenotype of an offspring. We are thus assuming that our measurements have been made on a scale for which the cultural effect of a parent is a linear function of the parental score.

We are also assuming that a scale can be found on which cultural effects do not interact with effects which contribute to variation in $x$. If variation in $x$ is genetic this amounts to an assumption of no genotype-environmental interaction. Alternatively, if $x$ varies for environmental reasons, we are assuming a scale can be found on which the effects of such specific environmental factors do not interact with those of culture. There is a variety of ways of specifying the contribution of such interactions, but more important, there are many methods by which the contribution of such interactions might be detected for a particular scale of measurement (see Jinks and Fulker, 1970). Cavalli-Sforza and Feldman (1973) incorporate genotypeenvironment interaction in their single gene model for cultural transmission by allowing the value of $b$ to depend on the genotype of the offspring concerned. In practice, it may be difficult to satisfy, simultaneously, all the manifold criteria of a desirable scale in which case a simple model may not be sufficient to account for all the measured variation.

The phenotype $p_{n i}$, has been written simply in terms of the non-cultural $\left(x_{n i}\right)$ effect expressed in generation $n$ and the cultural effect of the two parents in generation $n+1$. We may, however, define the parental phenotypes similarly in terms of their non-cultural components $\left(x_{n+1}, i j\right)$ and the cultural influence of their parents, and so on, ad infinitum. This means that the cultural effect on an individual in generation, $n=0$ say, can be expressed in terms of the non-cultural effects of all his ancestors.

Thus, we may write

$$
\begin{aligned}
p_{0 i}=\xi_{0}+x_{0 i}+2 b \xi_{1}+b \sum_{j=1}^{j=2} x_{1 i j}+4 b^{2} \xi_{2}+b^{2} & \sum_{j=1}^{j=4} x_{2 i j}+\ldots \\
& +(2 b)^{n} \xi_{n}+b^{n} \sum_{j=1}^{j=2^{n}} x_{n i j}+\ldots
\end{aligned}
$$

The $\xi$ 's are constants which appear in the model for every $p_{0 i}$. We may summarise the contribution of all these constants more simply by writing:

$$
p_{0 i}=c+x_{0 i}+b \sum_{j=1}^{j=2} x_{1 i j}+b^{2} \sum_{j=1}^{j=4} x_{2 i j}+\ldots b^{n} \sum_{j=1}^{j=2^{n}} x_{n i j}+\ldots
$$

where

$$
c=\xi_{0}+2 b \xi_{1}+4 b^{2} \xi_{2}+\ldots(2 b)^{n} \xi_{n}+\ldots .
$$

At equilibrium we may put $\xi_{1}=\xi_{2} \ldots=\xi_{n} \ldots=\xi_{e}$, so in the limit, as $n \rightarrow \infty$ we have, for $b<\frac{1}{2}$

$$
c=\frac{1}{1-2 b} \xi_{e}
$$

From (2) it is possible to obtain expectations for the variances and covariances of relatives directly, but it is a little easier to start in terms of (1) and write for the variance, $V_{p_{0}}$. of unrelated individuals in generation $n=0$ providing mating is random:

$$
V_{p_{0}}=V_{x_{0}}+2 b^{2} V_{p_{1}}+4 b W_{x_{0}, p_{1}}
$$


where $V_{x_{0}}$ is the variance of non-cultural effects in generation $n=0, V_{p_{1}}$ is the phenotypic variance of individuals in the parental generation, and $W_{x_{0}, p_{1}}$ is the covariance of phenotypic effects in the parental generation with non-cultural deviations in the current generation. If there is no change over generations in the factors which contribute to variation in $x$ and we have equilibrium for the total variance such that $V_{p}=V_{p_{0}}=V_{p_{1}} \ldots$

$$
V_{p}=\left(V_{x}+4 b W_{x_{0}, p_{1}}\right) /\left(1-2 b^{2}\right)
$$

Clearly, such an equilibrium can only exist in practice if $2 b^{2}<1$. So far, we have avoided any assumptions about the causes of variation in the non-cultural deviations, $x$. Now it is necessary since we wish to express $V_{x}$ and $W_{x_{0}, p_{1}}$ in more familiar terms. If $V_{x}$ can be attributed purely to chance environmental factors $\left(E_{1}\right.$, in the notation of biometrical genetics), then the covariance term $W_{x_{0}, p_{1}}$ is zero and we have:

$$
V_{p}=\frac{1}{1-2 b^{2}} E_{1} \text {. }
$$

Under these circumstances, the environmental variation between families is simply:

$$
\begin{aligned}
E_{2} & =V_{p}-E_{1} \\
& =\frac{2 b^{2}}{1-2 b^{2}} E_{1} .
\end{aligned}
$$

If $V_{x}$ is partly genetic, we no longer expect $W_{x_{0}, p_{1}}$ to be zero because there will be a genetic correlation between the cultural effects of parents and the non-cultural deviations of their offspring. This creates, for individuals reared by their natural parents, genotype-environmental covariation such that genetical differences between families are correlated with the cultural differences between them. The variance of individuals reared by their natural parents is expected to differ from that of individuals fostered at random by an amount equal to the genotype-environmental covariance term, $4 b W_{x_{0}, p_{1}}$. We may expand $W_{x_{0}, p_{1}}$ in series and put:

$$
W_{x_{0}, p_{1}}=W_{x_{0}, x_{1}}+2 b W_{x_{0}, x_{2}}+\ldots(2 b)^{n-1} W_{x_{0}, x_{n}} \cdots
$$

Now, when mating is random and when there are no epistatic interactions between loci, we have:

$$
W_{x_{0}, x_{k}}=\left(\frac{1}{2}\right)^{k} V_{A} .
$$

$V_{A}$ denotes the additive component of genetical variance for a randomly mating population (Falconer, 1960) and is equal to $\frac{1}{2} D_{R}$ in the biometrical genetical notation (Mather and Jinks, 1971). Our series thus becomes:

$$
W_{x_{0}, p_{1}}=\frac{1}{2} V_{A}\left(1+2 \cdot \frac{1}{2} \cdot b+4 \cdot \frac{1}{4} \cdot b^{2} \ldots+2^{n}\left(\frac{1}{2}\right)^{n} b^{n} \ldots\right)
$$

which, in the limit as $n \rightarrow \infty$ becomes:

$$
W_{x_{0}, p_{1}}=\frac{1}{2(1-b)} V_{A}
$$

By proceeding to the limit we have assumed that remote ancestors are not inbred. This assumption is unrealistic but distant relatives do not make a 
very big contribution when $b$ is less than about 0.5 . In the short-term assortative mating is likely to present far greater practical difficulties for our model.

When mating is random, therefore, and $V_{x}$ is wholly genetic we have, for the contribution of genotype-environmental covariance to the variation of individuals reared by their natural parents:

$$
4 b W_{x_{0}, p_{1}}=\frac{2 b}{(1-b)} V_{A} .
$$

In the absence of epistasis we may represent $V_{x}$ by the sum of the additive and dominance components of genetical variation $V_{A}$ and $V_{D}$ respectively. $V_{D}$ is equal to $\frac{1}{4} H_{R}$ in the notation of biometrical genetics (Mather and Jinks, 1971). Our expression (4) for the total variance thus becomes:

$$
\begin{aligned}
V_{p} & =\frac{1}{1-2 b^{2}}\left[\left(1+\frac{2 b}{1-b}\right) V_{A}+V_{D}\right] \\
& =\frac{1}{1-2 b^{2}}\left[\frac{1+b}{1-b} V_{A}+V_{D}\right] .
\end{aligned}
$$

When $b=0$, i.e. in the absence of cultural effects, this reduces to the familiar expression for the total genetic variance: $V_{p}=V_{x}=V_{A}+V_{D}=\frac{1}{2} D_{R}+\frac{1}{4} H_{R}$.

Given genetical variation in $x$ which is perpetuated culturally we can thus express our common environmental component $\left(E_{2}\right)$ and the covariance between cultural and genetical differences between families $\left(\mathrm{COV}_{2} e_{2}\right.$ in Jinks and Fulker's, 1970, erminology) in terms of familiar components of genetical variation, $V_{A}$ and $V_{D}$, and the coefficient, $b$, of cultural transmission. Thus we get:

$$
\begin{aligned}
& E_{2}=2 b^{2} V_{p} \text {, where } V_{p} \text { is defined as in (8) above. } \\
& 2 \operatorname{COV}_{g_{2}} e_{2}=4 b W_{x_{0}, p_{1}} \text {, defined as in (7) above. }
\end{aligned}
$$

If individuals are reared by randomly chosen foster parents the genotypeenvironmental component (7) does not appear in the expectation of the total variance which now reduces to

$$
\begin{aligned}
V_{p f} & =V_{x}+2 b^{2} V_{p} \\
& =V_{A}+V_{D}+2 b^{2} V_{p}
\end{aligned}
$$

the subscript " $f$ " distinguishing the variance of individuals reared by foster parents from that of individuals reared by their natural parents. We may also obtain the covariance of randomly adopted children with their foster parents from:

$$
W_{f_{p}}=b V_{p}
$$

where the definition of $V_{p}$ depends, as before, on the factors which contribute to $V_{x}$.

Our approach so far, shows that phenotypic variation arising due to environmental accidents or genetical segregation can be maintained and amplified (or dampened) by cultural transmission. We also see that empirical estimates of between-family environmental variance $\left(E_{2}\right)$ and of the covariance of genetical and environmental differences between families 
$\left(\mathrm{COV}_{2} e_{2}\right)$ do not satisfy the need for any more general causal explanation. We see, however, that variation which appears in an estimate of $E_{2}$ may ultimately be reflecting the cultural consequences of genetical variability in an ancestral generation. In Darlington's (1971) terms we have the possibility of a "genetic environment" which may itself be under the influence of natural selection.

If we detect $E_{2}$ without $\mathrm{COV}_{2} e_{2}$ then we would suspect that environmental differences between families are of a random non-cultural kind, or that the phenotypic variation which cultural transmission perpetuates is largely environmental. Should a study detect $\mathrm{COVg}_{2} e_{2}$, however, this would lend fairly strong support to the theoretical position that cultural transmission is a medium through which genetical differences are amplified or diminished. Positive genotype-environmental covariance would imply that cultural transmission is serving to augment individual differences. Negative genotype-environmental covariance would imply that the effect of cultural transmission is serving to moderate the consequences of genetical variation.

Should cultural transmission affect traits related to fitness we might expect selection to act not merely on individuals but kinships i.e. the phenotype of a parent may enhance or diminish his offspring's chance of survival. Several authors (e.g. Hamilton, 1964; Maynard-Smith, 1964) have considered mathematical aspects of kin selection. The important issue is the recognition that some kind of cultural transmission is an essential prerequisite of any selective mechanism which operates on families rather than individuals. If we could detect genotype-environmental covariance for a given trait this would imply the existence of the raw material on which kin selection could operate. The most likely data to support such a finding would be a comparison of the total variances of fostered and unfostered individuals (Jinks and Fulker, 1970; Cavalli-Sforza and Feldman, 1973). Such data are difficult to obtain and difficult to interpret unambiguously. Any findings from these studies would have to be consistent with data on the covariation of relatives, so we now examine the consequences of cultural transmission for the similarity of human relatives who have been measured on some continuously variable trait.

\section{The GOVARIANGes OF RELATIVES}

(a) Ancestral covariances

We may represent an ancestral covariance by $W_{p_{0}, p_{k}}$. This is the covariance between unrelated individuals in generation $n=0$ and their ancestors in the ancestral generation $n=k$. We take, for each $p_{0 i}$ one randomly chosen individual out of the $2^{k}$ ancestors in the $k$ th ancestral generation. The corresponding score of that ancestor is $p_{k i}$. When mating is random, only those ancestors of $p_{0 i}$ who are also descendants of $p_{k i}$ contribute to the cultural covariance of the $p_{0}$ and $p_{k}$. In every generation $n<k$ there is just one descendant of $p_{k i}$ who is also an ancestor of $p_{0 i}$. For example, only the paternal phenotype, and not the maternal phenotype may contribute to the cultural covariation of grandchildren and their paternal grandmothers.

We can now write:

$$
W_{p_{0}, p_{k}}=W_{x_{0}, p_{k}}+b W_{x_{11}} p_{k}+b^{2} W_{x_{2}, p_{k}}+\ldots b^{k-1} W_{x_{k-1}, p_{k}}+b^{k} V_{p} .
$$


If all variation in $x$ is due to chance environmental factors, then the covariance terms on the right-hand side are all expected to be zero. This leaves for a system in which culture transmits differences of environmental origin:

$$
\begin{aligned}
W_{p_{0}, p_{k}} & =b^{k} V_{p} \\
& =\frac{b^{k} E_{1}}{1-2 b^{2}} .
\end{aligned}
$$

If, however, some of the variation in $x$ is genetically determined, we no longer expect $W_{x_{0}, p_{k}}$ etc. to be zero because of the genetical correlation between individuals and their ancestors. We may obtain these covariances quite simply since, when mating is random:

$$
W_{x_{l}, p_{l+k}}=\left(\frac{1}{1-b}\right) \frac{1}{2^{k}} V_{A} .
$$

This follows, by applying the approach used in obtaining (7) above. So for our expectation for the ancestral covariance we have:

$$
\begin{aligned}
W_{p_{0}, p_{k}} & =\frac{1}{1-b} V_{A}\left[\left(\frac{1}{2}\right)^{k}+\left(\frac{1}{2}\right)^{k-1} b+\left(\frac{1}{2}\right)^{k-2} b^{2}+\ldots \frac{1}{2} b^{k-1}\right]+b^{k} V_{p} \\
& =\frac{1}{2^{k}(1-b)} V_{A}\left[1+2 b+4 b^{2}+\ldots+(2 b)^{k-1}\right]+b^{k} V_{p} .
\end{aligned}
$$

The finite series in square brackets simplifies thus:

$$
\left[1+2 b+\ldots+(2 b)^{k-1}\right]=\frac{1-(2 b)^{k}}{1-2 b} .
$$

We may substitute this in (11). When $V_{x}$ is wholly genetic we may write in our expectation for $V_{p}(8)$ and obtain:

$$
W_{p_{0}, p_{k}}=\frac{1-(2 b)^{k}}{2^{k}(1-b)(1-2 b)} V_{A}+\frac{b^{k}}{1-2 b^{2}}\left(\frac{1+b}{1-b} V_{A}+V_{D}\right)
$$

Clearly, if $V_{x}$ is due to both genetical and environmental factors we simply amend the expectation of $V_{p}$ accordingly and make the appropriate substitution in (11). In general, if our assumption of equilibrium is correct we may write our expectation for the ancestral covariances in terms of the coefficient, $b$, of cultural transmission, and the factors which contribute to variation in $x$.

(b) Covariances of twins, siblings and cousins

Jinks and Fulker (1970) give expectations of statistics from analyses of variance of twins and siblings reared together and apart in terms of the variances and covariances of genetical and environmental effects. We may obtain the expectations for these relatives in terms of our model simply by substituting for their empirical $E_{2}$ component the appropriate expectations on the basis of our model of cultural transmission (see above) and for their empirical covariance of genetical and environmental influence between families the corresponding theoretical expectation on the basis of our model. 
Jinks and Fulker already give the theoretical expectations of the genetical components of variation within and between twin and sibling families.

\section{(i) Trins and siblings}

The total variance of such individuals is given in general form by (4) above, but the precise expression will depend on our model for $V_{x}(5,8)$. As far as the cultural and genotype-environmental covariation components of covariance between individuals, twins and siblings will be equally alike in terms of our model since the contribution of these factors depends on the parental phenotype, not on the factors which contribute to non-cultural deviations. The genetical component of twin and sibling covariance, however, will depend on the degree of relationship. Following Jinks and Fulker (1970) we have, for the covariance of monozygotic twins reared together:

$$
W_{M Z T}=G_{1}+G_{2}+E_{2}+2 \mathrm{COVg}_{2} e_{2}
$$

and for

$$
W_{\text {Sib } T}=G_{2}+E_{2}+2 \mathrm{COVg}_{2} e_{2}
$$

where $G_{1}$ is the component of genetical variation within sibling families, due to segregation within the family, and $G_{2}$ is the component of genetical variation between families. When mating is random Jinks and Fulker point out that:

$$
\begin{aligned}
& G_{1}=\frac{1}{4} D_{R}+\frac{3}{16} H_{R} \\
& G_{2}=\frac{1}{4} D_{R}+\frac{1}{16} H_{R}
\end{aligned}
$$

in the notation of biometrical genetics. In Falconer's (1960) notation this means:

$$
\begin{aligned}
& G_{1}=\frac{1}{2} V_{A}+\frac{3}{4} V_{D} \\
& G_{2}=\frac{1}{2} V_{A}+\frac{1}{4} V_{D} .
\end{aligned}
$$

We have shown already how we may express the component of environmental variance between families and the genotype-environmental covariance component in terms of the cultural consequences of polygenic variation and chance environmental factors. We may substitute these expectations for the contributions of $E_{2}$ and $\mathrm{COV}_{2} e_{2}$ in (13) and (14) to specify the contribution of cultural effects to the covariance of monozygotic twins and siblings.

We thus obtain:

$$
W_{\mathrm{MZT}}=V_{A}+V_{D}+\frac{2 b}{1-b} V_{A}+2 b^{2} V_{p}
$$

and

$$
W_{\mathrm{Sib} T}=\frac{1}{2} V_{A}+\frac{1}{4} V_{D}+\frac{2 b}{1-b} V_{A}+2 b^{2} V_{p}
$$

$V_{p}$ is the expected population variance of unrelated individuals reared by their natural parents and depends on the nature of the factors which contribute to individual differences which are not themselves culturally determined. 
Thus $V_{p}$ may itself be written in terms of the coefficient of cultural transmission, $b$, the additive and dominance components of genetical variation $V_{A}$ and $V_{D}$ and the component of specific environmental variation within families $E_{1}$ (see equations 5 and 8 above).

\section{(ii) Cousins}

When we turn to more distant relatives it is impossible, as Jinks and Fulker point out, to obtain any satisfactory model in terms of purely empirical components like $G_{1}, G_{2}$ etc., since new parameters would be needed for each new statistic. This necessitates the adoption of the more general theoretical formulation of biometrical genetics in which the variances and covariances of relatives are specified in terms of the frequencies and effects of the genes which contribute to individual differences for a quantitative trait. In obtaining the expectations of covariances for cousins and more distant relatives, therefore, we choose once more to work in terms of $V_{A}, V_{D}\left(\frac{1}{2} D_{R}\right.$ and $\left.\frac{1}{4} H_{R}\right)$ etc.

We trace the ancestry of collaterals as far as the point where they share common ancestors. Thus we trace first cousins to their common grandparents. In writing the model for cousins we express their phenotypic deviations in terms of their own non-cultural effects and the cultural effects of their parents and grandparents. We put:

$$
\begin{aligned}
& p_{01 i}=\xi_{e}+x_{01 i}+b \sum_{j=1}^{j=2} x_{11 i j}+b^{2} \Sigma p_{21 i j} \\
& p_{02 i}=\xi_{e}+x_{02 i}+b \sum_{j=1}^{j=2} x_{12 i j}+b^{2} \Sigma p_{22 i j}
\end{aligned}
$$

$p_{01 i}$ and $p_{02 i}$ represent the phenotypic scores of the first and second individuals of the $i$ th cousin pair. The $x_{0}$ refer to their non-cultural deviations and the $x_{1}$ to the non-cultural deviations of their parents. The phenotypic scores of their grandparents are given by the $p_{2}$ elements.

One parent of $p_{01 i}$ is a sibling of one parent of $p_{02 i}$ so their cultural effects will be correlated. Two of the grandparents of one cousin are also grandparents of the other, so their cultural effects will contribute to the cousin covariance. Writing $W_{\text {Cous }}$ for the cousin covariance we have:

$$
\begin{aligned}
W_{\text {Cous }}=W_{p_{01}, p_{02}}=W_{x_{01}, x_{02}} & +b\left(W_{x_{01}, x_{12}}+W_{x_{02}, x_{11}}\right) \\
& +b^{2} W_{x_{12}, x_{11}}+4 b^{2} W_{x_{0}, p_{2}}+4 b^{3} W_{x_{1}, p_{2}}+2 b^{4} V_{p} .
\end{aligned}
$$

When all variation perpetuated culturally is created initially by chance all the covariance terms in the expectation of the cousin covariance are expected to be zero leaving:

$$
W_{\text {Cous }}=2 b^{4} V_{p}=\frac{2 b^{4} E_{1}}{1-2 b^{2}} .
$$

If, however, there is a genetical component in $V_{x}$ this is no longer the case. We now have:

$$
\begin{aligned}
& W_{x_{01}, x_{02}}=\frac{1}{8} V_{A}=\frac{1}{16} D_{R} \quad \text { (the genetical covariance of cousins); } \\
& W_{x_{01}, x_{12}}=W_{x_{02}, x_{11}}=\frac{1}{4} V_{A}=\frac{1}{8} D_{R} \quad \text { (the avuncular genetical covariance) }
\end{aligned}
$$




$$
\begin{aligned}
& W_{x_{11}, x_{12}}=\frac{1}{2} V_{A}+\frac{1}{4} V_{D}=\frac{1}{4} D_{R}+\frac{1}{16} H_{R} \text { (the genetical covariance of full } \\
& \text { siblings) } \\
& W_{x_{1}, p_{2}}=\frac{1}{2(1-b)} V_{A}
\end{aligned}
$$

and

$$
W_{x_{0}, p_{2}}=\frac{1}{2^{2}(1-b)} V_{A} .
$$

Our expectation now becomes:

$$
\begin{aligned}
W_{\text {Cous }} & =V_{A}\left[\frac{1}{8}+\frac{1}{2} b+\frac{1}{2} b^{2}+\left(\frac{1}{1-b}\right)\left(b^{2}+2 b^{3}\right)\right]+\frac{1}{4} b^{2} V_{D}+2 b^{4} V_{p} \\
& =(1+2 b) V_{A}\left[\frac{1}{8}(1+2 b)+\frac{b^{2}}{1-b}\right]+\frac{1}{4} b^{2} V_{D}+\frac{2 b^{4}}{1-2 b^{2}}\left[\frac{(1+b)}{1-b} V_{A}+\frac{1}{2} V_{D}\right]
\end{aligned}
$$

given that there is no random environmental variation in $x$.

\section{Discussion}

So far we have regarded the environmental and genetical origin of culturally transmitted variation as distinct possibilities. In all probability the observed variation will depend on both. We have considered the effects of genetical polymorphism separately from those of random environmental effects. Providing we can regard all non-cultural environmental factors as random we may obtain the expectations for the variances and covariances of individuals in a randomly mating population simply by combining the expectations under a simple genetical model for the origin of culturally transmitted differences with those which assume a simple environmental basis for their origin.

In table 1 we give the expectations for several statistics which might be obtained from randomly mating populations given that both genetical factors and random environmental factors were contributing to culturally inherited differences. Although the expectations look unmanageable, experience with the fitting of non-linear models in biometrical genetics (e.g. Eaves, 1975) suggests that if data on sufficient degrees of relatives can be obtained it should be possible to obtain approximate maximum-likelihood estimates of the parameters of such a model and to see if they provide an adequate explanation of individual differences for a given trait. In the absence of cultural transmission (i.e. when $b=0$ ) the expectations of table 1 simplify to those applicable to a randomly mating population in the absence of common environmental effects.

Our expectations in table 1 suggest the kinds of data that might be used to detect the presence of cultural effects. It can be seen, as many writers have shown, that data on fostered individuals provide the most simple basis for the detection of $E_{2}$ effects. Jinks and Fulker (1970) also commented that such data are virtually essential if we are to detect the covariation of genetical and environmental deviations of the kind which we see here to be generated by the cultural transmission of inherited differences. Cavalli-Sforza and Feldman (1973) also remark on the significance of data on fostered individuals 


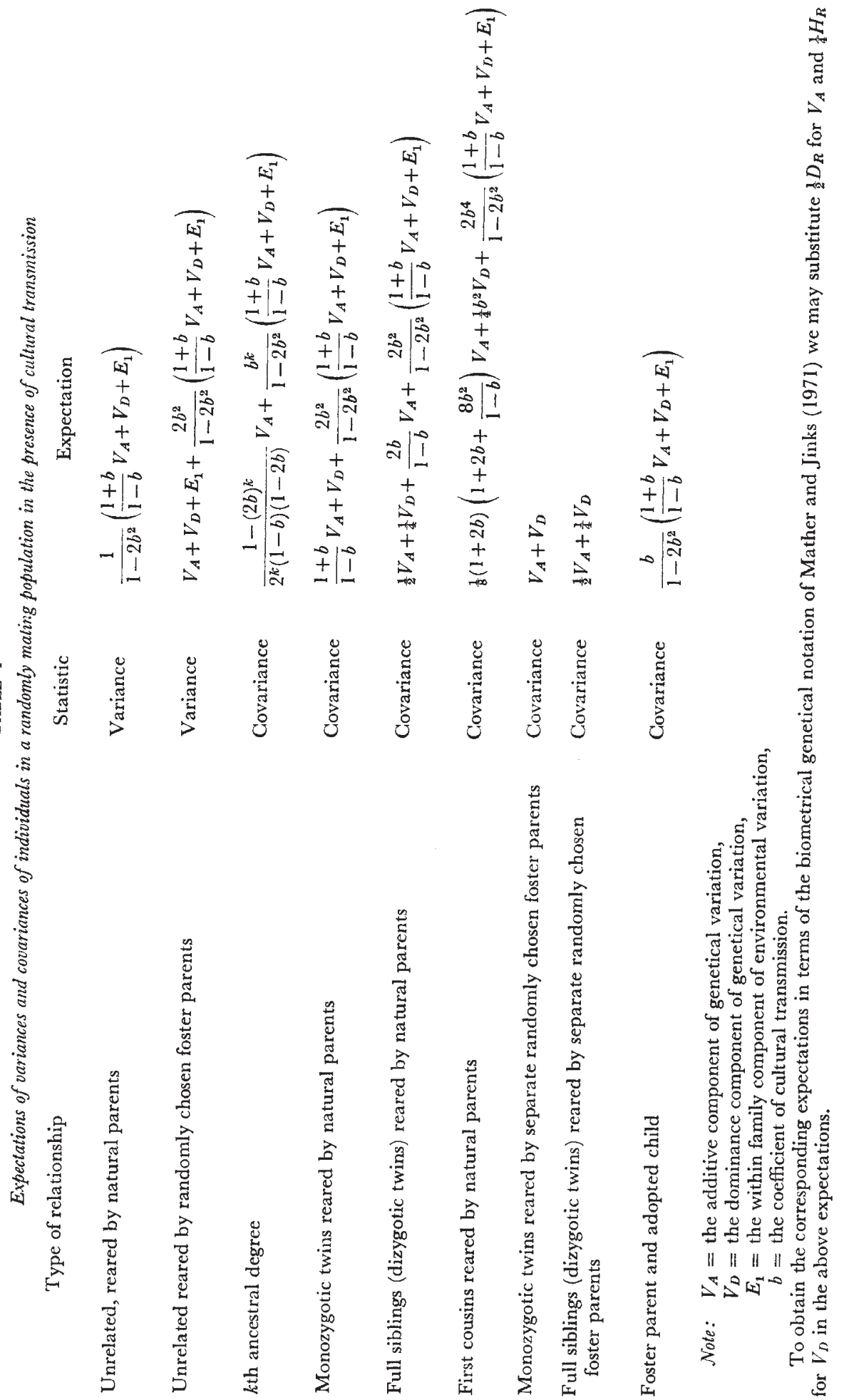


for the detection of cultural transmission but do not relate the components of their particular model to common environmental effects or to the components of genotype-environmental covariation specified by other workers.

The advantage of the current approach lies in the fact that a single parameter, $b$, might under some circumstances, be sufficient to explain the cultural components of similarity between many kinds of relatives and thus avoid the need to fit many purely empirical parameters to account for the family environment and genotype-environment covariation.

In order to give some insight into the consequences of cultural transmission for the pattern of individual differences in a population table 2 has been prepared. It shows the expected numerical values of various variances and covariances between relatives for different degrees of cultural transmission

TABLE 2

Population variances and covariances under different degrees of cultural transmission for a randomly mating population in which environmental differences depend entirely on additive genetical variation

\begin{tabular}{|c|c|c|c|c|c|c|c|c|}
\hline$b$ & $V_{p}$ & $V_{p_{f}}$ & $E_{2}$ & $2 \mathrm{COV}_{g_{2} e_{2}}$ & $W_{p}$ & $W_{g_{p}}$ & $W_{g g_{p}}$ & $W_{f_{p}}$ \\
\hline$-0 \cdot 70$ & $8 \cdot 824$ & $9 \cdot 647$ & $8 \cdot 647$ & -0.824 & $-5 \cdot 882$ & $4 \cdot 265$ & -2.912 & $-6 \cdot 157$ \\
\hline-0.60 & 0.893 & $1 \cdot 643$ & 0.643 & -0.750 & -0.223 & $0 \cdot 290$ & -0.096 & -0.537 \\
\hline-0.50 & 0.667 & 1.333 & 0.333 & -0.667 & 0.000 & $0 \cdot 167$ & $0 \cdot 000$ & -0.333 \\
\hline-0.36 & 0.635 & $1 \cdot 165$ & $0 \cdot 165$ & -0.529 & $0 \cdot 139$ & $0 \cdot 134$ & 0.044 & -0.229 \\
\hline-0.20 & 0.725 & $1 \cdot 058$ & 0.058 & -0.333 & $0 \cdot 272$ & $0 \cdot 154$ & 0.074 & -0.145 \\
\hline$-0 \cdot 10$ & 0.835 & $1 \cdot 017$ & 0.017 & -0.182 & 0.371 & $0 \cdot 190$ & 0.095 & -0.084 \\
\hline 0.00 & $1 \cdot 000$ & $1 \cdot 000$ & 0.000 & 0.000 & $0 \cdot 500$ & $0 \cdot 250$ & $0 \cdot 125$ & $0 \cdot 000$ \\
\hline $0 \cdot 10$ & $1 \cdot 247$ & $1 \cdot 025$ & 0.025 & 0.222 & 0.680 & $0 \cdot 346$ & $0 \cdot 173$ & 0.125 \\
\hline $0 \cdot 20$ & 1.630 & $1 \cdot 130$ & $0 \cdot 130$ & 0.500 & 0.951 & 0.503 & $0 \cdot 257$ & 0.326 \\
\hline 0.36 & $2 \cdot 869$ & $1 \cdot 744$ & 0.744 & $1 \cdot 125$ & $1 \cdot 814$ & $1 \cdot 044$ & 0.571 & $1 \cdot 033$ \\
\hline 0.48 & 5.278 & $3 \cdot 432$ & 0.432 & 1.846 & 3.495 & $2 \cdot 158$ & $1 \cdot 276$ & $2 \cdot 533$ \\
\hline
\end{tabular}

Key: $\quad b=$ coefficient of cultural transmission,

$V_{p}=$ phenotypic variance of individuals reared by natural parents,

$V_{p_{f}}=$ phenotypic variance of individuals reared by randomly chosen foster parents,

$E_{\mathbf{2}}=$ environmental variance between families,

$2 \mathrm{COV}_{g_{2} e_{2}}=$ covariance of environmental and genetical differences between families,

$W_{p}=$ covariance with natural parent,

$W_{g_{p}}=$ covariance with grandparent,

$W_{g g_{p}}=$ covariance with great-grandparent,

$W_{f_{p}}=$ covariance with foster parent.

Note: The additive genetical component is fixed at unity throughout.

(i.e. for different values of $b$ ) in a population in which all the variation is due ultimately to the additive effects of many independent loci. We have assumed a value of unity for the additive genetical component of variance (i.e. $V_{A}=\frac{1}{2} D_{R}=1$ ).

The values of $b$ have been selected from the range $-\sqrt{\frac{1}{2}}<b<\frac{1}{2}$ within which an equilibrium may occur. The figures show how cultural transmission can greatly increase the phenotypic variance and thus greatly magnify apparent genetical differences, when $b$ is positive. When $b$ is negative the phenotypic variance is reduced except as $b$ approaches the limit for the occurrence of equilibrium. Genotype environmental covariance produces $V_{p}>V_{p f}$ when $b$ is positive, and $V_{p}<V_{p f}$ when $b$ is negative. Comparison of the values of these two variances, a likely basis for any test of genotype-environmental covariance, shows that even in this most extreme 
instance of complete genetical determination of cultural differences the variance ratio cannot exceed the value of 2 attained for the ratio $F=V_{p f} / V_{p}$ when $b=-0 \cdot 5$. The ratio is generally smaller than this, suggesting that quite large samples of fostered individuals are going to be required if we are going to reject the hypothesis of zero genotype-environmental covariance for any but the largest cultural effects.

Examination of the ancestral covariances, including those for foster parents and their foster children reveals some of the many additional possibilities for the similarities and differences between relatives which can be created by cultural transmission. We see that cultural transmission will

TABLE 3

Population variances and covariances under different degrees of cultural transmission for a randomly mating population in which cultural differences depend entirely on random environmental variation

\begin{tabular}{ccrrrrrr}
$b$ & \multicolumn{1}{c}{$V_{p}$} & \multicolumn{1}{c}{$V_{p_{f}}$} & \multicolumn{1}{c}{$E_{2}$} & \multicolumn{1}{c}{$W_{p}$} & \multicolumn{1}{c}{$W_{g_{p}}$} & \multicolumn{1}{c}{$W_{g g_{p}}$} & \multicolumn{1}{c}{$W_{f_{p}}$} \\
-0.70 & 50.000 & 50.000 & 49.000 & -35.000 & 24.500 & -17.150 & -35.000 \\
-0.60 & 3.571 & 3.571 & 2.751 & -2.143 & 1.286 & -0.771 & -2.143 \\
-0.50 & 2.000 & 2.000 & 1.000 & -1.000 & 0.500 & -0.250 & -1.000 \\
-0.36 & 1.350 & 1.350 & 0.350 & -0.486 & 0.175 & -0.063 & -0.486 \\
-0.20 & 1.087 & 1.087 & 0.087 & -0.217 & 0.043 & -0.009 & -0.217 \\
-0.10 & 1.020 & 1.020 & 0.020 & -0.102 & 0.010 & -0.001 & -0.102 \\
0.00 & 1.000 & 1.000 & 0.000 & $0 \cdot 000$ & 0.000 & 0.000 & 0.000 \\
0.10 & 1.020 & 1.020 & 0.020 & 0.102 & 0.010 & 0.001 & 0.102 \\
0.20 & 1.087 & 1.087 & 0.087 & 0.217 & 0.043 & 0.009 & 0.217 \\
0.36 & 1.350 & 1.350 & 0.350 & 0.486 & 0.175 & 0.063 & 0.486 \\
0.48 & 1.855 & 1.855 & 0.855 & 0.890 & 0.427 & 0.205 & 0.890
\end{tabular}

Key: as for Table 2.

Note: The variance due to random environmental effects is fixed at unity throughout.

increase the parent-offspring correlation when $b$ is positive and, therefore, mimic some of the effects of assortative mating. Assortative mating by itself, however, will not produce the non-zero covariance expected for foster parents and their adopted children. A large negative coefficient of cultural transmission is expected to produce some quite bizarre results, including grand-parental correlations in excess of those for parents and offspring. Although it is possible for such systems to exist in theory it is difficult to see how they could evolve in practice since for any trait related to fitness we may imagine that fitter parents are likely to improve rather than diminish their offspring's chances of survival (i.e. $b$ is expected to be positive).

For comparison, table 3 gives values expected for some of the same variances and covariances when cultural transmission operates on individual differences whose ultimate origins lie in random environmental effects. Obviously, since there is no genetical variation there can be no genotypeenvironmental covariation and the variance of individuals no longer depends on whether they are reared by natural or foster parents. In addition the parent-offspring covariances are the same, irrespective of whether individuals are reared by natural or foster parents. In the absence of genetical variation it is quite impossible for the signs of the two kinds of parent-offspring covariance to differ. This is, however, possible for negative $b$ values when culture transmits information based on genetical differences between parents (cf. table 2).

The two tables represent opposite extremes for the origin of culturally 
transmissible differences. In all probability both genetical and environmental influences may have cultural consequences. In principle, the various parameters of this (or similar models) are estimable although large samples are likely to be needed to produce precise estimates. Clearly, such a model may not fit particular data, but if it did the gain in economy and insight would be substantial, because most of the significant consequences of cultural transmission might be expressed in a single parameter, $b$.

We see that, under some circumstances, the problem of cultural transmission can be cast in a quantitative form which specifies explicitly the consequences of cultural inheritance for individual differences. Of course, this does not pretend to be an exhaustive treatment of the issue. There may well be different assumptions that we would wish to make in generating a mathematical model for human variation in the presence of cultural transmission. The important point is that as long as the discussion of culturally maintained variability avoids the explicit formulation of a mathematical model it will not be testable in practice and will have little scientific value. We see that recognising the possibility of cultural transmission does not avoid the difficulty of finding the origin of those differences which culture perpetuates. Such differences may reflect genetical polymorphism or environmental heterogeneity and in all probability involve both.

The strength of this model lies in our ability to specify the assumptions. We have assumed:

1. Gultural transmission operates chiefly by the influence of parents on their offspring;

2. There is no interaction between the effects of culture and the noncultural effects on individual behaviour;

3. Mating is random;

4. The population is in equilibrium;

5. The cultural effect of parents on their offspring is linearly related to the parental phenotype and is constant over generations;

6. In so far as genes contribute to the variation they are many, autosomal, and do not display epistatic interactions;

7. Parents do not interact in determining the cultural environment of their offspring.

Some of these assumptions are more critical than others. If two assumptions were to be selected as most critical we would probably consider those relating to the mating system and cultural equilibrium. It is likely that mating is assortative for many culturally significant traits. Assortative mating clearly will tend to inflate any cultural influences on the similarity between relatives even in the absence of genetical variation. It would be premature to claim that a mathematical treatment of cultural transmission in the presence of assortative mating would be impossible, but it is likely to be tedious and require still further assumptions about gene action if it is to be reasonably tractable.

Whether or not the population can safely be regarded as being in equilibrium is likely to depend on the kind of trait which is measured. If we find, in practice, a trait for which the variance is increasing from one generation to the next one simple explanation will be that cultural transmission is super-efficient in that cultural advantages and disadvantages, rather than being just "maintained" from one generation to the next are actually 
being amplified. Such effects might be further magnified by any increase in the amount of assortative mating.

The consequences of cultural transmission are, in many respects, like those of assortative mating in that both tend to make distant relatives (such as cousins) considerably more alike than we would expect on the basis of a simple system in which neither was present. Often the data are likely to be ambiguous but marked similarity between distant relatives, without a substantial marital correlation would clearly require explanation in cultural terms. In so far as cultural transmission contributes to the common environmental $\left(E_{2}\right)$ component of individuals reared together the most powerful tests for its effects are those best able to detect $E_{2}$ and will involve collection of data on individuals reared by foster parents. Many writers have discussed the theoretical and practical problems of detecting $E_{2}$ (e.g. Newman, Freeman and Holzinger, 1937; Cattell, 1960; Jinks and Fulker, 1970; Eaves, 1972; Cavalli-Sforza and Feldman, 1973).

We should detect cultural inheritance initially as an $E_{2}$ effect, whether cultural transmission is responsible for maintaining differences which are genetic or environmental. When, however, the cultural differences are due partly to genetical differences we expect, in addition, significant covariation of genetical effects and cultural differences $\left(\mathrm{COV}_{2} e_{2}\right)$. The most direct and powerful test of such effects is the comparison of the total variances of individuals reared by their natural parents with those reared by foster parents.

The question remains "What traits are likely to show cultural transmission?" Much effort was expended in the early days of testing to ensure that measurements of "I.Q." measured "innate" ability. This might imply that such ability measures were the wrong target for analyses of cultural variation. The evidence to hand, whatever its weaknesses does not lend much support to the view that cultural heterogeneity, whether of hereditary or environmental origin, contributes greatly to variation in I.Q. There is, however, every reason to suppose that traits which are less "refined" should reflect the consequences of cultural and social inequality and show quite a different picture for the causes of variation. Jinks and Fulker (1970) found that Burt's published correlations between relatives for educational attainments were not consistent with a simple genotype-environmental model and suggested that covariation between genetical deviations and those differences contributing to environmental variation between families could provide one explanation of this finding. The implication of their suggestion is clear: the present social environment is such that cultural transmission is not merely partly responsible for maintaining individual differences in attainment, but is perpetuating over future generations the cultural consequences of genetical variation among their ancestors. Hewitt (1974) in a detailed re-analysis of twin data published by Eaves and Eysenck (1974) concluded that " radicalism" scores on a social attitudes questionnaire displayed a pattern of transmission characteristic of a simple additive genotype-environmental system in which the variation between families was inflated by assortative mating, cultural effects or both. These findings, based as they are on fairly crude data, are none the less suggestive that cultural transmission could be relevant to the understanding of variation in some human traits. It is of great theoretical interest to human biology, and possibly of some social concern, to define and measure those aspects of 
human behaviour which are most affected by cultural transmission particularly where culture is perpetuating and exaggerating the less desirable aspects of genetical polymorphism. The approach outlined here, though not exhaustive, may lend some hope that the problem of analysing cultural variation is more manageable than is sometimes believed.

Acknowledgments.-This work is part of a research programme in Psychogenetics supported by the MRC. I am grateful to Professor J. L. Jinks, Professor K. Mather, Dr J. S. Gale, Krystyna Last and Nick Martin for their helpful comments.

\section{REFERENCES}

CATTELL, R. B. 1960. The multiple variance analysis equations and solutions for naturenurture research on continuous variables. Psychological Review, 67, 353-372.

GAVAlLI-SFORZA, L. L., AND FELDMAN, M. W. 1973. Cultural versus biological inheritance: phenotypic transmission from parents to children (a theory of the effect of parental phenotypes on children's phenotypes). Am. F. Human Genet., 25, 618-637.

DARLINGtON, C. D. 1971. Axiom and process in genetics. Nature, 234, 521-525.

EAVES, L. J. 1972. Computer simulation of sample size and experimental design in human psychogenetics. Psych. Bull. 77, 144-152.

EAVES, L. J. 1973. Assortative mating and intelligence: an analysis of pedigree data. Heredity, 30, 199-210.

EAVES, L. J. 1975. Testing models for variation in intelligence. Heredity, 34, 132-136.

EAVES, L. J., AND EYSENGK, H. J. 1974. Genetics and the development of social attitudes. Nature, 249, 288-289.

EAVES, L. J., AND EYSENCK, H. J. 1975. The nature of extraversion: a genetical analysis. Fournal of personality and social psychology, 32, 102-112.

FALCONER, D. S. 1960. Introduction to Quantitative Genetics. Oliver and Boyd, Edinburgh.

hamilton, w. D. 1964. The genetical evolution of social behaviour. I. F. Theoret. Biol., 7, $1-16$.

HEWrTr, J. K. 1974. An analysis of data from a twin study of social attitudes. M.Sc. Thesis, University of Birmingham.

JENcks, c. 1973. Inequality: A Reassessment of the Effects of Family and Schooling in America. Allen Lane, London.

JENSEN, A. R. 1974. Kinship correlations reported by Sir Cyril Burt. Behavior. Genetics, 4, 128.

JINKS, J. L., AND FULKER, D. W. 1970. A comparison of the biometrical genetical MAVA and classical approaches to the analysis of human behaviour. Psych. Bull., 73, 311-349.

mather, K., AND Jinks, J. L. 1971. Biometrical Genetics. Second edition. Chapman \& Hall, London.

MAYNARD-SMITH, J. 1964. Group selection and kin selection. Nature, 201, 1145-1147.

NEWMAN, H. H., FREeman, F. N., AND Holzinger, K. J. 1937. Twins, a Study of Heredity and Environment. University of Chicago Press. 